\title{
Teeth implant design using weighted sum multi-objective function for topology optimization and real coding genetic algorithm
}

\author{
Musaddiq Al Ali ${ }^{\mathrm{a},}$, Amjad Y. Sahib ${ }^{\mathrm{b}}$, Muazez Al Alic \\ ${ }^{\text {a }}$ Hiroshima University, 1-3-2 Kagamiyama, Higashi-Hiroshima City, 739-8511, Japan \\ bUniversity of Wasit, Wasit Province, Hay Al Rabee, Kut, Iraq \\ ${ }^{c}$ Al-Ayen University, Thi Qar Province, Niel St., Nasiriyah, Iraq \\ *Corresponding Author: mosadeq007@yahoo.com
}

\begin{abstract}
Teeth implant suffeers from stress sheildin and mismatch of bio-mechanical properties, such as weight and stifness. Multi-objective optimization. In order to design better implant, topology optimization of multi-objective function is used in this work. Weighted sum functions are offer a single function to be addressed and optimized which ease finding extremum and feasible design for the various functions that make this single multi-objective function. In this paper, a genetic algorithm is used to determine optimal weights of the three-objective function that used, which are minimizing compliance, minimizing local stress, and minimizing norm function of local stress. This multi-objective function is used to design the optimal shape of dental implant for minimal stress shielding in the jaw. Stress shielding problem is solved by designing the implant's compliance to match the compliance of the original missing tooth. two stress minimization function has been examined, which is local stress and norm function. Conditions of the design was matching the stiffness of implant which made from titanium with original replaced tooth and setting volume fraction of topology optimization to be $40 \%$ of original design problem to offer weight matching. Results shows feasible design. Strain based fatigue simulation shows considerably low difference between non-optimized implant and optimized implant.
\end{abstract}

Keywords: Genetic algorithm, Topology optimization, Multi-objective function, Stress minimization, Implant, Fatigue.

\section{Introduction}

Generally, to get the valid mechanical design for the certain application, practically there are several conflicting aspects to be considered. For example, global minimum compliance of a design domain is not associated with minimum stress field distribution. To get a solution for which, the design variables and constraint, satisfy the design criterion in the best way of minimum triage of all needed or desired aspects; multi-objective optimization gives such opportunity. Our goal in this paper is to give design procedure of a multiobjective function by identifying best weights of the sum function based on shape and design target, using genetic algorithm; such multi-objective function will be used in Topographic based optimization design methods. First, Topographic optimization based design method such as shape optimization and topology optimization is being increasingly used to achieve a feasible design with pure mathematical logic. i.e. find maximum or minimum of the certain function design for the desired application. Multi constraint objective function (MCOF) is used for these design method, however, it needs to calculate the complete model for each design step. Adding to that the concept itself can move from global extremum of the design application to a local global extremum of the localized objective function, which can be local minima. For example; design high real structure should meet at least two criterion which is stiffer, and less stress concentration areas structure. Stiffness base objective function (OF) can't avoid stress concentration part due to the nature of the $\mathrm{OF}$ which is the minimization of compliance $\left(\mathbf{F}^{\mathrm{T}} \mathbf{U}\right)$. Adding stress constraint will limit maximum allowable stress of the produced design, but it is 
not assuring that it is the best Practical design. As mentioned previously, the multi-objective function can give a solution for such problem. Considering topology optimization, Heuristic technique as optimization methodology. Nodal forces and displacement, are used which needs considerably large computational power Metaheuristic techniques are adopted in many types of research to be a directly addressed criterion, underlying sets of objective and constraint within. Genetic algorithm, (GA) is a highly regarded method to solve the structural optimization problem. However, its effectiveness; its efficiency degrades with increasing numbers of constraint to be satisfied. The direct use of GA is not applicable for large number of element problem, for topology optimization such that, practically, topography to be optimized are complicated in nature, making finding valid design with orthodox method a difficult task. Multi-objective optimization in general form can be GA optimization target. Weighted functions ${ }^{(1)}$ is used as topology optimization objective function. ${ }^{(2)}$ Selection of weights for is vital for global optimality to be satisfied. GA is adopted as global optimization solver to select the best weighting for sum multi-objective function. It can be selected using an evolutionary algorithm with predetermined sets by initialization of GA. The real coding genetic algorithm was used. GA is used successfully for the more complex problem, yet reasonable constraint numbers ${ }^{(3)}$.

Sum weighted multi-objective function (MOF) is used in engineering design to join multiple different design aspect, such as direct economic cost, stiffness, electric conductivity, etc. (4) It is used strictly as a tool, which means, for some researchers, it is a matter of trial and error and closed-loop feedback to set the appropriate weights. Of course, the initial estimation is based on the functions themselves. MOO weight selection and optimization is a challenging problem. The set of weight after setting usually be a reference for another researcher works. So, it will be tested and then generalized for their functional extremum. Without having sufficient background about what and how and why weighted MOO been set in general, and for the certain application, the time cost of the computation can be so high. Weighted MOO was investigated by many researchers in terms of how to set, and how to solve. Zedah ${ }^{(5)}$ adopt Pareto optimality as the way of solving positive weighted method sum MOO. Koski et al ${ }^{(6)}$ introduce norm method to scale the class of MOO. They applied the weight systematic change in the weights while minimizing the volume and the nodal displacement of the simple truss structure. Kin et al ${ }^{(7)}$ used adaptive weighted sum method approach by adapting Pareto front. To achieve feasible mechanical design for the certain application, practically there are several conflicting aspects to be considered. For example, global minimum compliance of a design domain is not associated with minimum stress field distribution. To locate a solution for which, the design variables and constraint, satisfy the design criterion in the best way of minimum triage of all needed or desired aspects; multi-objective optimization gives such opportunity. Heuristic and metaheuristic methods were intensively used. Metaheuristic methods, such as genetic algorithm (GA) and particle swarm optimization try to mimic biological activities. They prove their competitiveness with another optimality criterion. Yet, they lose their edge with increasing design variables. The direct use of GA (3) is questionable for topology optimization such that, practically, topography to be optimized are complicated in nature, making finding a valid design with the orthodox method a challenging task. Multi-objective optimization (1) in general form can be GA optimization target. The genetic algorithm has been used in design composite structures. Riche and Haftka studied stacking sequence optimization of the thin composite plate ${ }^{(8)}$. Liu et al studied stacking sequences of predetermining fiber orientations for maximizing buckling load ${ }^{(9)}$. Park et al, used Finite element analysis as a numerical solution to address stacking sequence and orientation considering moderate thick plates (considering first order shear deformation theory), optimizing the plate using a genetic algorithm ${ }^{(10)}$. Farshi and Herasati implemented Tsai-Hill criterion, to develop optimality criterion based optimization technique for layer thickness and orientation ${ }^{(11)}$. Topology optimization has been developed rapidly in last decades, and still a considerable attractive topic to be addressed due to free computer design. It based on the based auto design in order to find the optimal shape of the designed part based on objective subjected to the constraint. Topology optimization (TO) be designed method based on a mathematical scheme to introduce non-existence material within design space to get designer properties such as lightweight or certain mechanical properties. Topology optimization has been developed from Maxwell dilemma of optimal weight truss design, by Michell(12). Himp (13) studied strain field by adopting slip line, which at that time was regarded as an important aspect of plastic deformation implementations. Drucker et al ${ }^{(14)}$ applied constant dissipation per unit volume as their study to stress-strain fields and strain energy. Chan (15) study the optimization of static stability of truss structure by developing a technique to determine topographic based strain filed. Charrett and Rozvany ${ }^{(16)}$ adopted Prager - shield 
implementation in order to find optimal design criterion considering rigid-perfectly plastic systems under multiple loading. Rozvany and Prager ${ }^{(17)}$ studied optimal design of grillage like continua. Their approach was spatial distribution within confined grillage units. Rossow and Taylor ${ }^{(18)}$ used finite element method as a numerical solution to find the optimum thickness of variable thickness sheets. Potential energy for the elastic sheet in-plane stress assumption was addressed. By introducing holes into plate structure, this work founded shape optimization. Cheng and Olhoff ${ }^{(19)}$ implement finite element method as a numerical solution to optimize the thickness of annual plate with stiffened like approach. Bendsoe ${ }^{(20)}$ studied Solid Isotropic Material with Penalization (SIMP) based on Taylors work.

\section{Topology optimization}

\subsection{Compliance and Stress based objective functions}

Solid Isotropic Material with Penalization (SIMP) is a scheme apply for discretized design domain to find smooth optimal structure in which, material properties set to be constant for the discretized domain. ${ }^{(21,22)}$ however, the existence of building block which is set in what so-called density function composed of material existence $\rho$ of conditioned power multiply by mechanical properties.

$$
\begin{aligned}
& \lim _{\text {volf } \rightarrow 0} x\left\{\Gamma\left(\Omega \cap \Omega_{\text {mat }}=0\right)\right\} \\
& \text { Extermum. } \Psi(\rho) \mid E_{\min \rightarrow 0}+x^{q}\left(E_{\min \rightarrow 0}+E_{\text {mat } \rightarrow 1}\right) \\
& \text { s.t. } \quad \int_{\Omega_{d}} \rho d x \leq V_{d}, 0<x_{\min }<x_{e}<1 \quad \forall x \in \Omega_{d}
\end{aligned}
$$

Where $\Psi$, is objective function.

Power (q)that satisfy condition of two dimensions ${ }^{(22)}$ set to be within

$$
\left.q \in] 15 \frac{1-v}{7-5 v}, \frac{3-3 v}{2+4 v}\right]
$$

\section{Fixation}

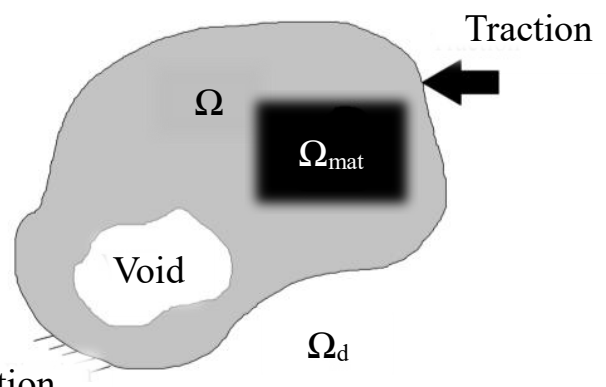

Fig. 1. Topology optimization Problem
The solution in the scope of current scheme face some challenges as checkerboards and Nonexistence. The latter is Mesh dependency problem, so Nonexistence tends to introduce non-existence element, severely, to satisfy solution with decreasing value of objective function. To solve it, relaxation principal is introduced, modifying density function with what so-called gray region ${ }^{(23)}(0<\rho<1)$. As well as gray area, heuristic searching with sufficient constraint will be a remedy. Checkerboards is also a byproduct of discretization. The solution in it tends to be non-applicable due to mesh dependency. One of an effective solution uses high order elements, however, computation resources and time will easily be consumed under pressure of real-life complexity of design domain. Another solution is filtered independence filtering process. One of which, Sigmund ${ }^{(22)}$ was introduced

$$
\frac{\partial f^{\prime}(\rho)}{\partial x_{e}}=\frac{1}{x_{e} \sum_{n=1}^{N}\left(r_{\min }-d(e, n)\right)} \sum_{n=1}^{N}\left(r_{\min }-d(e, r)\right) x_{n} \frac{\partial f(\rho)}{\partial x_{e}}
$$

Where $d(e, r)$ is distance between central element xe and each neighbor elements $\mathrm{x}(\mathrm{n}=1 \rightarrow \mathrm{N})$. The produced modified sensitivity will update the designated Optimally criteria.

Compliance objective function is aimed to maximize stiffness of the structure by minimizing the compliance

Despite the fact that compliance based topology optimization leads to the stiffer structure by minimization; by design, stress consideration is poor for such objective function. Stress is sensitive toward confined topography. In the case of sharp corners within the structure, stress increase dramatically with corner sharpness. To address stress issue in a general view, finite elements should be chosen for the high susceptible parts. In the case of topology optimization; theoretically, the design should be done by the chosen optimization algorithm. In such case, the prior identification of susceptible parts is not a practice issue. To solve this matter, stress should be addressed in the optimization problem. In this work, three ways were addressed, local stress based objective function, aggregated objective function. Within two-dimensional design domain, stresses represented as two normal, and shear stresses. However, stress tensor is noted giving an estimation of stress state that makes a failure. Theories of elastic failure are the key to determining stress states that permitted for maximum estimated structural life. Von-Mises (equation (4)) is widely accepted for a metallic structure with certain ductility materials.

$$
\sigma_{\mathrm{vms}}=\sqrt[2]{\sigma_{\mathrm{x}}^{2}+\sigma_{\mathrm{y}}^{2}-\sigma_{\mathrm{x}} \sigma_{\mathrm{y}}+3 \tau_{\mathrm{xy}}^{2}}
$$


Yield criterion is the envelope that design domain stays within. The maximum allowable stress could be identifying for certain material. Singularity is problem face topology optimization ${ }^{(24)}$. In order to establish stress criterion as a valid objective function to be extremum, the relationship of scaled stress should be formed to satisfying the following; simplicity to decrease unnecessary commotions, physical coherence, and address material discretization directly. qpapproach is satisfying the above; localized stress ${ }^{(2,24,25)}$ or some paper named it microscopic stress ${ }^{(25)}$ which take the form

$$
\rho^{\mathrm{p}-\mathrm{q}}\left(\frac{\sigma_{\mathrm{vms}}}{\sigma_{\text {yield }}}\right)
$$

Aggregative methods such as p-norm ${ }^{(26)}$ are used to introduce a global stress objective function. To solve stress state, finite element method is the common effective way. Discretization using FEM is adopted in topology optimization to get the design. Stress arises vast constraint number, which degrades solution with increasing resolution of it (i.e. increase element numbers). Such partial differential equation set with vast number of constraint can be considered within Lebesgue space ${ }^{(27,28)}$

$$
\ell^{\wp}(\Omega)=\left\{\sigma_{\mathrm{vms}} \in \mathcal{M}(\Omega): \int_{\Omega}\left|\sigma_{\mathrm{vms}}(\mathrm{t})\right|^{\wp} \mathrm{dt}\right\}
$$

With norm defined by

$$
\left\|\sigma_{\mathrm{vms}}\right\|_{\wp}=\left\{\begin{array}{c}
\sqrt[\wp]{\ell^{\wp}(\Omega)}, 1 \leq \mathrm{p}>\infty \\
\sup \left\{\left|\sigma_{\mathrm{vms}}\right| 1 \leq \wp<\sigma_{\mathrm{vms}}\right\}, \quad \wp=\infty
\end{array}\right.
$$

Optimization will consider the first part of norm Equation (7). $\wp$, which consider as stress norm parameter control the tendency of converging for the optimization process. The effect of normative power $(\wp)$ can be shown in figure 2. In case (A), $\quad \sigma_{\mathrm{vms}}$ tend to be the average value, while increasing $\wp$ value shift norm to a higher value $(\wp \rightarrow \infty \Rightarrow$ $\left.\left\|\sigma_{\mathrm{vms}}\right\| \rightarrow \max \left(\sigma_{\mathrm{vms}}\right)\right)$. Another major problem in stress as the topology optimization design based target is the locality nature of such target ${ }^{(24)}$. According to the field in discretized design domain; stress criterion strongly varies by design part. In order to decrees the severity of locality problem, scaling target to maximum value $\sigma_{\text {yield }}$ such that

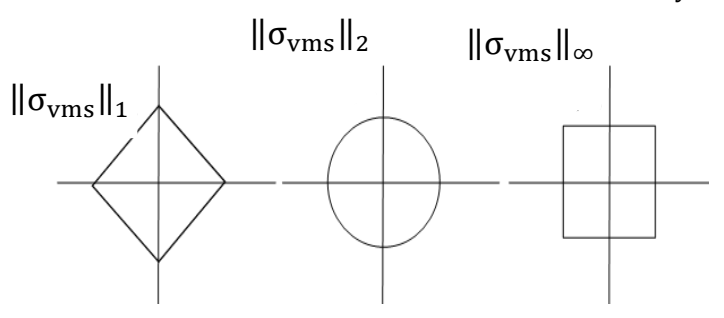

Fig. 2. Pnorm function envelops with various powers. $\frac{\sigma_{\mathrm{vms}}}{\sigma_{\text {yield }}}<1 \in \Gamma$.The objective function and boundaries will take the form

$$
\left\|\sigma_{\text {von_Mises }}\right\|_{\wp}=\left(\sum_{\mathrm{i}=1}^{\mathrm{n}}\left|\frac{\sigma_{\text {von_Mises }}}{\sigma_{\text {yield }}}\right|^{\wp}\right)^{\wp^{-1}}
$$

Where $\wp$ normative power

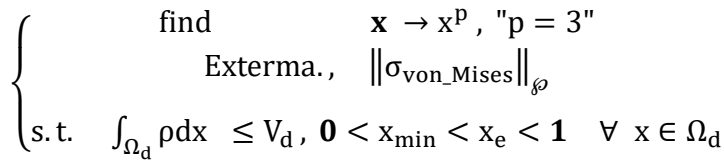

$\left\|\sigma_{\text {von_Mises }}\right\|_{\mathbf{p}}$ is wildly know as P-norm function ${ }^{(29,30)}$ or sometimes KK function ${ }^{(29)}$ refining to Park K. and Kikuchi. Associated with appropriate constraint, sensitivity analysis plays major role in achieving converging results with minimizing computational and time efforts. First order sensitivity analysis is required be perform each iteration. Adjoin variable method is used to develop a unified formulation for representing response variation in term of variation design. Aggregation objective function which subjected to constraint with respect to design variable x. Due to discretization nature of TO, mesh quality and type play vital role in pre-and post-processing of design. As mentioned previously, SBTO affected by FEM accumulative analysis history. High order elements may increase the odds "theoretically "of better design, and higher resolution designs as well as increasing element geometric density. However, computational and time cost may be a serious problem along with convergence. Topology optimization in terms of sensitivity analysis approaches needs derivative to apply an iterative Lagrangian multiplier. As Heuristic and meta-heuristic methods are used to extremum functions for the field of optimization, heuristic methods, as Lagrangian is implemented in many topology optimization types of research, showing its effectiveness and simplicity to apply and control. Adding to that, metaheuristics showed in some techniques weakness toward increasing design variables number. Some research's value advancing of quantum advancing in quantum-based searching approaches and advancing in metaheuristic hyperdilation, but in the scope of near future, heuristic searching proved to be as a keystone in global optimization of many variables as topology optimization. Global search algorithms need sensitivity analysis. In such case, derivative might contain certain difficulties, especially, the derivative should be in term of design variables. First order sensitivity analysis is required be perform each iteration. The adjoint variable method is used to develop a unified formulation for representing 
response variation in term of variation design. Aggregation objective function which subjected to a constraint with respect to design variable $x_{e}$. finite element based sensitivity analysis using adjoint method gives

$$
\frac{\mathrm{d} \Psi}{\mathrm{dx}_{\mathrm{e}}}=\lambda^{\mathrm{T}}\left(\frac{\mathrm{dF}}{\mathrm{dx}_{\mathrm{e}}}-\frac{\mathrm{dK}}{\mathrm{dx}_{\mathrm{e}}} \mathrm{U}\right)+\frac{\mathrm{d} \Psi}{\mathrm{dx}_{\mathrm{e}}}
$$

Here, $\Psi$ is a density-related function to be derivative. $\mathrm{K}$ is stiffness matrix,

$$
K=\int_{V}[\mathrm{~B}]^{\mathrm{T}}[\mathrm{E}][\mathrm{B}] \mathrm{dV}
$$

Here $\lambda$ is the adjoint variable, which can be calculated from Eq.(10)

$$
\mathrm{K}^{\mathrm{T}} \lambda=\frac{\partial \Psi}{\partial \mathrm{U}}
$$

\subsection{Multi-objective function for structural optimization examples}

Multi-objective topology optimization by weighted sum function combined compliance and stress state-based function $^{(2)}$. Compliance minimization approach is giving the optimize stiffer structure. However, stress localization is a problem that row compliance function cannot address. Several conditional optimizations approach been introduced $^{(31)}$. However, stress based function noticed to normalized singularities efficiently. For stress based function, aggregative stress gives a smooth function, avoiding singularities, however, for microscopic(localized) stress, a minimum stress can be achieved in some cases. For example, comparing the topology optimizations cases that were shown in Figure (3)

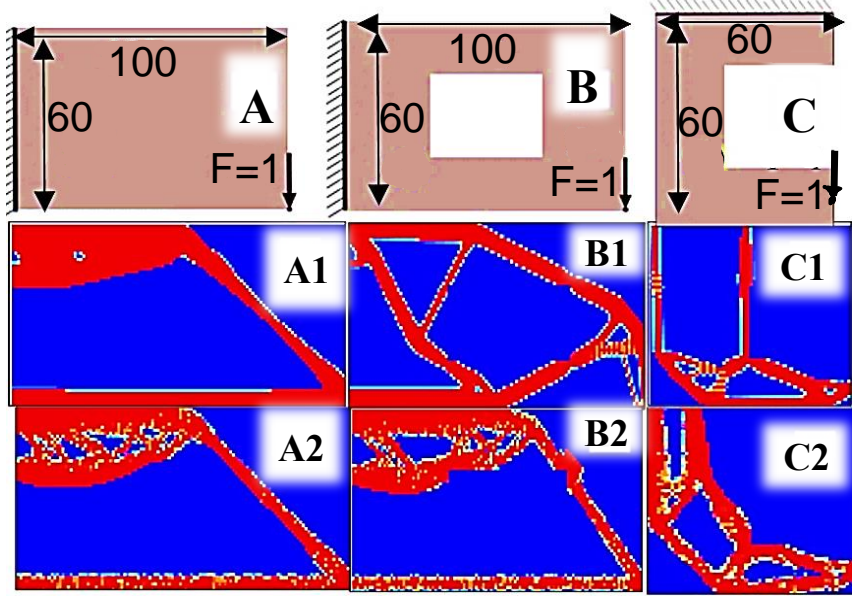

Fig. 3. Topology optimization example using stress objectives: A, B, C are topology problems and boundaries. A1, B1, C1 are local stress optimized structures. A2, B2, C3 are pnorm optimized structures.
Table 1. Von mises stress results of optimization examples.

\begin{tabular}{|l|l|l|l|}
\hline Structure & A & B & C \\
\hline Local Stress & 1.5232 & 1.4775 & 1.53 \\
\hline pnorm & 1.4651 & 1.8719 & 1.7598 \\
\hline
\end{tabular}

This test shows the non-conformity of stress prediction for a different type of structures due to numerical truncation of finite element. The element type that used in this example is four nodes rectangular element. Introducing second-order element and or changing element type improve the dealing with stress. Add to that, associated multiple types of an element such as bar element along with $2 \mathrm{~d}$ element (in the present case), also improve stress study, however, with increasing complexity of the structure, non-conformity appears. According to these previous examples, a multiobjective function of the three optimization approaches is been adopted will take the form in equation (13)

$$
M O O=w_{1} C+w_{2} L S+w_{3} P N
$$

Where, $\mathrm{w}_{1}, \mathrm{w}_{2}$ and $\mathrm{w}_{3}$ are weighting, $\mathrm{C}$ is compliance function, LS is local stress function, and $\mathrm{PN}$ is norm function. For Three $2 \mathrm{~d}$ cases have been studied: cantilever, a cantilever with a square hole, and L-shape (same as in Figure 3). The problem is a mimic to medical orthopedic design. In orthopedic design, the stress of the structure should be minimum as possible in order to increase fatigue life. In the same time, and for biological reasons stress shielding ${ }^{(32)}$ for example; the compliance of the structure should be so close to the part to be replaced. Usually, the biological part has a less young modulus of elasticity compared to orthopedic materials. Such problem has two contradictory parts which are compliance maximization while stress should minimize. For each stress based objective functions (Local stress and pnorm objective functions) their own cones and pores. As shown in Table. 1 sometimes stress is minimized better for local stress function than for pnorm and sometimes it is otherwise. This is due to the numerical discretization effect so; first order square element is used. Switching to higher order element will dramatically increase the computational time, and there is no guarantee, to get the global optima for single stress based objective function. In this, both approaches of stress objective function were used. In this paper, Young modulus of the design domain is 1 , and the Young modulus of the conditioned compliance materials is 0.6. The design procedure is shown in Figure (4). Topology optimization function which is used as GA objective function 
is using equation (13). The algorithm of TO is shown in Figure (5). Using MATLAB 2017, topology optimization and a genetic algorithm were written. The optimal weights that result for the three cases (Figure (6)) is shown in Table. 2

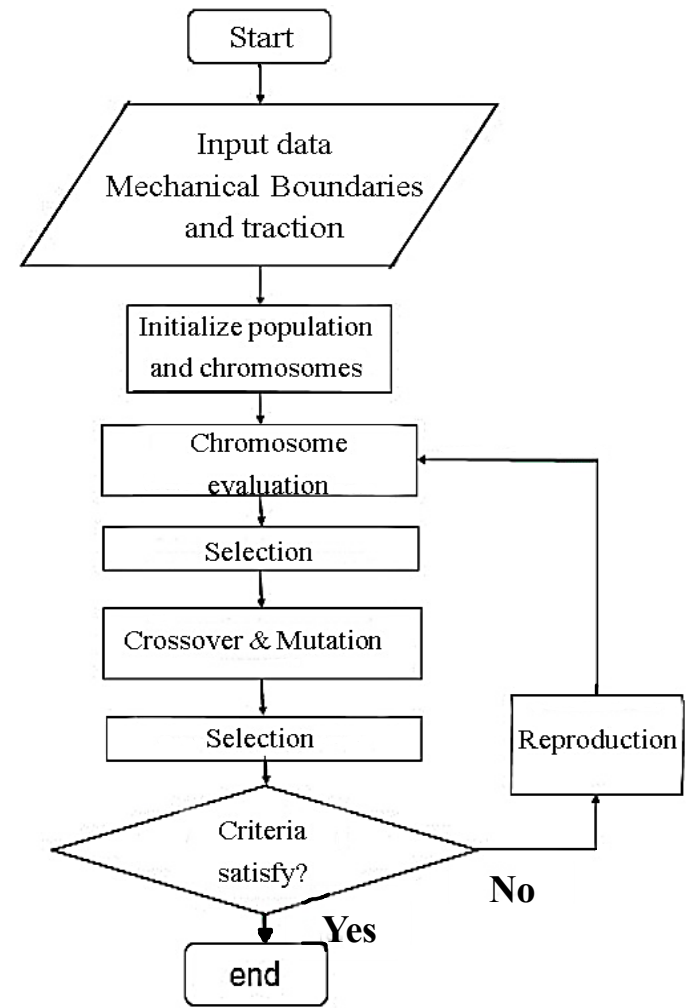

Fig. 4. GA optimization.

Table 2. optimal weights for the sum function obtained by GA.

\begin{tabular}{|l|l|l|l|}
\hline Structure & W1 & W2 & W3 \\
\hline A & 0.073752 & 0.065256 & 0.025745 \\
\hline B & 0.07585 & 0.051507 & 0.081343 \\
\hline C & 0.028496 & 0.053755 & 0.30189 \\
\hline
\end{tabular}

Table 3. optimal weights for the sum function obtained by GA.

\begin{tabular}{|l|l|}
\hline Structure & Maximum Von misses \\
\hline A & 1.4689 \\
\hline B & 1.5115 \\
\hline C & 2.54 \\
\hline
\end{tabular}

Table 4. Young modulus of elasticity (E) of dental materials

\begin{tabular}{|l|l|l|l|}
\hline Material & Tooth & Jaw & Titanium implant \\
\hline $\mathrm{E}(\mathrm{MPa})$ & 80.53 & 7.4 & 114 \\
\hline
\end{tabular}

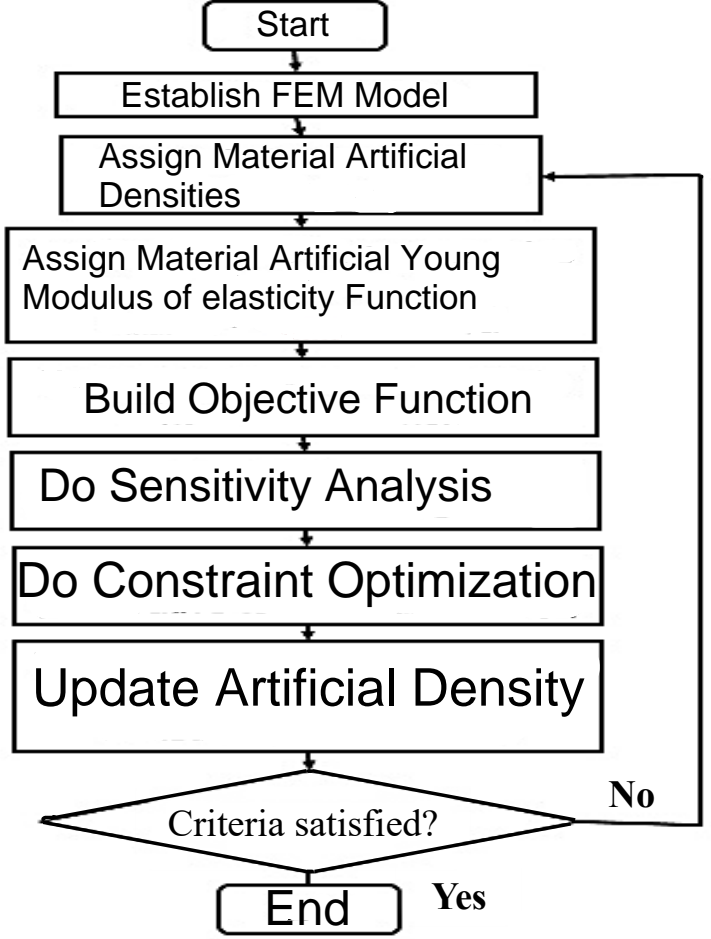

Fig. 5. Topology optimization Algorithm.

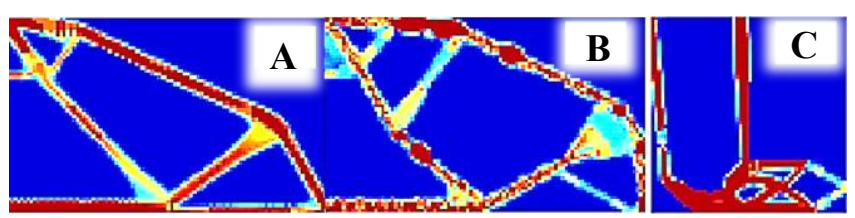

Fig. 6. GA - Topology optimization example using MOF for cases A, B, C

\section{Case study- Tooth implant design (Prime molar case)}

Here we adopted MOF design criterion to design optimal tooth implant, which matches the replaced tooth stiffness and has inside minimal stress concentration spots. Mechanical properties of the problem ${ }^{(33-35)}$ are shown in table 4. Force is chosen to be normal to tooth of $175 \mathrm{~N}$ magnitude The first step is calculating original tooth compliance for the loading case shown (as in Figure 7), and it was $\left(8.45 \times 10^{-1}\right)$. $3 \mathrm{~d}$ model was build using first-order tetrahedron element, with 12 degree of freedom.

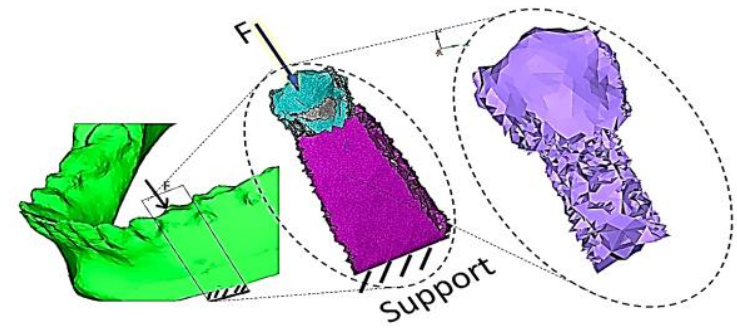

Fig. 7. Tooth model. 


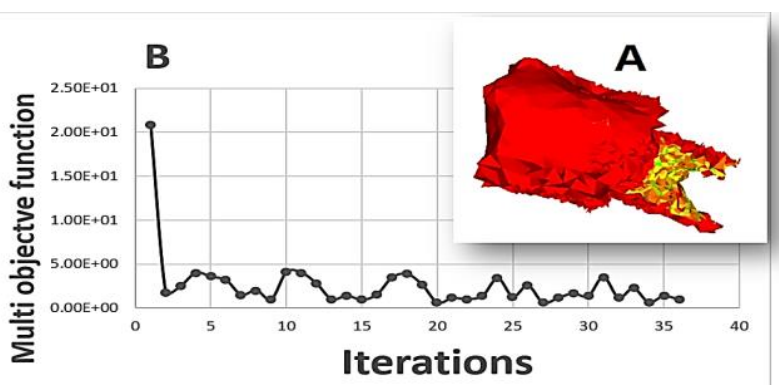

Fig. 7. Topology optimization results (A) Final tooth design, (B) Objective function history.

Genetic Algorithm gives the weights for multi-objective function (equation 13), shown in Table 5

Table 5. Optimal weights for the sum function obtained

\begin{tabular}{|l|l|l|}
\multicolumn{2}{c}{ for the implant } \\
\hline W1 & W2 & W3 \\
\hline 0.0064385 & 0.30092 & 0.47147 \\
\hline
\end{tabular}

With setting compliance of the implant to be the same as the missing original tooth, and reducing the volume fraction to $40 \%$ of original implant volume; Final topology optimization result shown in figure (8)

Reducing the volume fraction will match the weight of the implant to match one of the original teeth for better biomedical compatibility. A fatigue life estimation was performed for the designed prosthesis. The strain-life method was adopted for its accuracy and suitability for the loading conditions. Loading variation was considered as full revisers of $8 \%$ of the overall maximum strain, linear variated within the simulated biting time. Modeling of the strain-life is calculated as in equation (14).

$$
\varepsilon_{a}=0.01538157\left(2 N_{f}\right)^{-0.095}+0.35\left(2 N_{f}\right)^{-0.69}
$$

Simulation is done using OptiStruct solver, the crack starts to affect $5 \%$ of the total area after $\left(1.25 \times 10^{6}\right)$ cycle. This is what be set as fatigue life expectation for optimum design. Topology optimized implant shows less fatigue life $\left(6.712 \times 10^{5}\right)$.

\section{Conclusions}

In this paper, Topology optimization with multiobjective function was successfully done to design materials of high modulus of elasticity to match the stiffness of replacing material of lower modulus of elasticity. Biomechanical matching is achieved by matching stiffness and weight of produced design with the original missing part. Genetic algorithm gave weights specific to the problem. Stress minimization with the designed MOF gave considerable good fatigue life comparing to the original implant, taking into consideration that the optimized implant has better bio-medical compatibility, which limit the possibility of implant rejection. However the process of achieving MMO using genetic algorithm is taking more time with complex problem, the final implant shape is like the real original teeth, which can limit stress shielding and increase the life of the implant inside patient Jaw.

\section{References}

(1) R. T. Marler and J. S. Arora, "The weighted sum method for multi-objective optimization: new insights," Structural and multidisciplinary optimization, vol. 41, pp. 853-862, 2010.

(2) R. Yang and C. Chen, "Stress-based topology optimization," Structural optimization, vol. 12, pp. 98-105, 1996.

(3) A. Blanco, M. Delgado, and M. C. Pegalajar, "A real-coded genetic algorithm for training recurrent neural networks," Neural networks, vol. 14, pp. 93105, 2001.

(4) S. A. Kassaimah, A.-A. M. Mohamed, and F. A. Kolkailah, "Bi-criteria optimum design of laminated plates under uniform load and shear," Diversity into the next century, pp. 731-737, 1995.

(5) L. Zadeh, "Optimality and non-scalar-valued performance criteria," IEEE transactions on Automatic Control, vol. 8, pp. 59-60, 1963.

(6) J. Koski and R. Silvennoinen, "Norm methods and partial weighting in multicriterion optimization of structures," International Journal for Numerical Methods in Engineering, vol. 24, pp. 1101-1121, 1987.

(7) I. Y. Kim and O. De Weck, "Adaptive weighted sum method for multiobjective optimization: a new method for Pareto front generation," Structural and multidisciplinary optimization, vol. 31, pp. 105-116, 2006.

(8) R. Le Riche and R. T. Haftka, "Optimization of laminate stacking sequence for buckling load maximization by genetic algorithm," AIAA journal, vol. 31, pp. 951-956, 1993.

(9) B. Liu, R. T. Haftka, M. A. Akgün, and A. Todoroki, "Permutation genetic algorithm for stacking 
sequence design of composite laminates," Computer methods in applied mechanics and engineering, vol. 186, pp. 357-372, 2000.

(10) J. Park, J. Hwang, C. Lee, and W. Hwang, "Stacking sequence design of composite laminates for maximum strength using genetic algorithms," Composite Structures, vol. 52, pp. 217-231, 2001.

(11) B. Farshi and S. Herasati, "Optimum weight design of fiber composite plates in flexure based on a two level strategy," Composite structures, vol. 73, pp. 495-504, 2006.

(12) A. G. M. Michell, "LVIII. The limits of economy of material in frame-structures," The London, Edinburgh, and Dublin Philosophical Magazine and Journal of Science, vol. 8, pp. 589-597, 1904.

(13) W. S. Hemp, "Theory of structural design," College of Aeronautics Cranfield1958.

(14) D. C. Drucker and R. Shield, "Design for Minimum Weight," BROWN UNIV PROVIDENCE RI1956.

(15) A. Chan, "The design of Michell optimum structures," College of Aeronautics Cranfield1960.

(16) D. Charrett and G. Rozvany, "Extensions of the Prager-Shield theory of optimal plastic design," International Journal of Non-Linear Mechanics, vol. 7, pp. 51-64, 1972.

(17) G. Rozvany and W. Prager, "Optimal design of partially discretized grillages," Journal of the Mechanics and Physics of Solids, vol. 24, pp. 125136, 1976.

(18) M. Rossow and J. Taylor, "A finite element method for the optimal design of variable thickness sheets," Aiaa Journal, vol. 11, pp. 1566-1569, 1973.

(19) K.-T. Cheng and N. Olhoff, "An investigation concerning optimal design of solid elastic plates," International Journal of Solids and Structures, vol. 17, pp. 305-323, 1981.

(20) M. P. Bendsøe, "Optimal shape design as a material distribution problem," Structural and multidisciplinary optimization, vol. 1, pp. 193-202, 1989.

(21) M. P. Bendsøe and O. Sigmund, "Material interpolation schemes in topology optimization," Archive of applied mechanics, vol. 69, pp. 635-654, 1999.

(22) M. P. Bendsøe, O. Sigmund, M. P. Bendsøe, and O. Sigmund, Topology optimization by distribution of isotropic material: Springer, 2004.
O. Sigmund, "A 99 line topology optimization code

written in Matlab," Structural and multidisciplinary optimization, vol. 21, pp. 120-127, 2001. 